Résumés des conférences et travaux

\title{
Histoire de l'architecture et des jardins du Japon prémoderne
}

(Milieu du XVI ${ }^{\mathrm{e}}$ siècle-milieu du XIX ${ }^{\mathrm{e}}$ siècle)

Nicolas Fiévé

\section{(2) OpenEdition}

Journals

Édition électronique

URL : https://journals.openedition.org/ashp/1066

DOI : 10.4000/ashp.1066

ISSN : 1969-6310

Éditeur

Publications de l'École Pratique des Hautes Études

\section{Édition imprimée}

Date de publication : 2 février 2011

Pagination : 350-354

ISSN : 0766-0677

\section{Référence électronique}

Nicolas Fiévé, "Histoire de l'architecture et des jardins du Japon prémoderne », Annuaire de l'École pratique des hautes études (EPHE), Section des sciences historiques et philologiques [En ligne], 141 | 2011, mis en ligne le 25 février 2011, consulté le 06 juillet 2021. URL : http://journals.openedition.org/ashp/ 1066 ; DOI : https://doi.org/10.4000/ashp.1066 


\title{
HISTOIRE DE L'ARCHITECTURE ET DES JARDINS DU JAPON PRÉMODERNE
}

\section{(MILIEU DU XVI ${ }^{\mathrm{e}}$ SIÈCLE-MILIEU DU XIX ${ }^{\mathrm{e}}$ SIÈCLE)}

\author{
Directeur d'études : M. Nicolas FIÉvÉ
}

Programme de l'année 2008-2009 : Urbanisme et paysage urbain de Kyôto, la capitale impériale. Première partie : aux origines du paysage urbain.

Depuis la fondation de Heian, au viII ${ }^{\mathrm{e}}$ siècle, l'urbanisme et l'architecture de la capitale impériale ont entretenu une relation privilégiée avec l'environnement naturel et végétal. L'espace urbain de Heian, aujourd'hui nommée Kyôto, a été organisé en relation étroite avec la nature sauvage présente sur le pourtour montagneux du bassin de Kyôto, alors que l'espace domestique a été conçu en relation constante avec la nature artificielle des jardins et des parcs, dont l'usage se généralise très tôt, dès le $\mathrm{IX}^{\mathrm{e}}$ siècle. La capitale impériale a ainsi été le lieu d'une organisation singulière de l'espace, fondée sur des liens originaux de l'homme avec la nature. À travers ces liens, les habitants de la capitale ont élaboré tout au long de l'histoire de la ville des ensembles paysagers, organisés aujourd'hui encore selon des systèmes uniques d'interaction entre l'homme et son environnement naturel. À l'intérieur de ces ensembles, ce sont aussi la morphologie des quartiers, l'ordonnancement de groupes d'édifices historiques, l'organisation spatiale de l'habitat domestique et des jardins, l'architecture des palais, des monastères bouddhiques et des sanctuaires shintô, qui témoignent de ces évolutions historiques et paysagères.

Afin de sensibiliser les auditeurs à une approche de la ville japonaise fondée sur les liens de l'homme à la nature, la conférence a présenté une analyse critique des systèmes paysagers représentatifs de phases successives de l'histoire humaine de la cité, qu'il s'agisse de paysages clairement définis par leur forme, leur lieu et leur histoire, ou de paysages évolutifs ou culturels, représentatifs d'une diversité souvent méconnue. Remontant aux origines de la capitale, les conférences de l'année 20082009 ont été consacrées à l'urbanisme ancien de Heian et, selon la perspective que nous venons d'évoquer, aux rapports entre les sites sacrés du pourtour montagneux, la géographie naturelle du site et la conception de l'espace urbain.

Contrairement à la Chine, l'Antiquité japonaise n'a pas connu de villes fortifiées. Heian n'échappe pas à cette règle. Dans cette vaste capitale au plan rectangulaire, les limites physiques de l'agglomération étaient celles des quatre dernières grandes avenues qui la bordaient à l'est, à l'ouest, au nord et au sud. À bien des égards, pourtant, la césure entre l'intérieur et l'extérieur de la ville relevait davantage de frontières conceptuelles et immatérielles héritées de l'animisme, mais aussi du bouddhisme et de la doctrine de la Voie du Yin et du Yang, Onmyôdô 陰陽道. Selon l'ordre impérial de 794 mentionné dans le Nihon kiryaku 日本紀略 (Annales abrégées du Japon), il était favorable de construire une capitale dans un 
pays «entouré de montagnes et de rivières ». Cette sagesse dans le choix du lieu était l'héritage de la pensée chinoise relative à l'établissement d'une capitale, ce que suggère l'édit impérial de transfert de la capitale lorsqu'il évoque le site en disant qu'il «s'accorde au motif des Quatre oiseaux », shikin gyôzu 四禽叶図, et qu'il « est abrité au pied des Trois montagnes », sanzan sakuchin 三山作鎮 (Shoku Nihongi 続日本紀, fasc. 4 : 33). Le choix du site remplissait les critères d'adéquation du lieu aux Quatre divinités, shishin 四神, figures centrales de la topomancie chinoise, symboles des quatre secteurs stellaires et de l'espace, auxquelles quatre sites naturels du bassin de Kyôto furent associés. Quant à la topographie remarquable des Trois montagnes, un archétype de l'urbanisme ancien du Yamato que l'on retrouve déjà dans l'ordonnancement de l'ancienne Nara (710-784), elle fut matérialisée par les collines Funaoka 船岡山, Kagura 神楽山, Narabi-ga-oka 並々岡, trois sites funéraires et sacrés depuis la préhistoire. Ces éminences naturelles dominaient le palais impérial sur ses faces nord, est et ouest, et les concepteurs de Heian les adoptèrent comme points de base du tracé urbain : l'axe nord-sud de la grande avenue Suzaku 朱雀大路 se situait dans l'alignement du sommet de Funaoka, alors que la Première grande avenue, Ichijô-ôji 一条大路, qui marquait la limite nord du palais impérial et de la capitale, était tracée dans l'axe est-ouest des collines Kagura et Narabi-ga-oka, ellesmêmes situées de part et d'autre de la ville. La grille protectrice des Quatre dieux et celle des Trois montagnes étaient en outre superposées à celle d'un autre ensemble, connu sous le nom des Quatre sièges de pierre, yon iwakura 四岩座. Selon les fudoki 風土記 (Récits du vent et de la terre), des annales régionales rédigées sous l'ordre de l'impératrice Genmei 元明天皇 (r. 707-715) à partir de 713, les théophanies se faisaient le plus souvent par le truchement d'un intermédiaire, comme un bloc rocheux, iwakura 岩座 (siège de pierre), un réceptacle naturel destiné à accueillir une divinité, situé sur une montagne ou dans une forêt sacrée, dite kamunabi ou kannabi 神名備 (pour accueillir la divinité). Plusieurs sanctuaires du bassin de Kyôto ont conservé des aspects de ces cultes anciens. Les Quatre sièges de pierre gardiens de la capitale se dressent aujourd'hui encore sur le mont Dainichi 大日山, à l'est de Kyôto au sanctuaire Himukai jinja 日向神社, dans le temple Konzôji 金蔵寺 à Ôharano 大原野 (à l'ouest), sur la montagne de Shiun 紫雲山, à proximité du sanctuaire Yamazumi jinja 山住神社 (au nord), et au pavillon de Fudôdô 不動堂 du temple Myôô.in 明王, au sud du bassin de Kyôto. Selon une citation du Konzôji ryaku engi 金蔵寺略 延喜 (Récit abrégé de la fondation du temple Konzôji, texte cité dans le Yôshûfushi 雍州府志 [Chroniques du Yamashiro, préface de 1684]), des écritures bouddhiques auraient été enfouies près d'un bloc rocheux sacré en vue de protéger la capitale impériale au moment de son transfert, sento 遷都 (Yôshûfushi, fasc. $5: 341$ ). La tradition transmise par le temple Fudôdô rapporte qu'au moment de la fondation de Kyôto, le moine Kûkai 空海 (774-835) aurait sculpté dans la pierre la statue d'un bouddha pour la disposer sur la colline boisée non loin des bâtiments. À l'entrée du temple, on peut encore lire sur le panneau frontal l'épigraphe Minami iwakura yama 南岩倉山 (montagne-siège de pierre-du-sud). Chacune des Trois montagnes protectrices du palais impérial étaient - et sont encore de nos jours - des kamunabi yama 神名備山, des montagnes pour accueillir la divinité. 
Autour de ces sites se déroulent d'anciennes formes de célébrations des divinités qui nous renseignent sur les anciennes conceptions de protection de la capitale. Sur la montagne de l'Homme, Otoko yama 男山, on trouve un «siège de pierre-du-sud», minami iwakura 南岩座山. Lorsqu'on trace sur une carte de Kyôto une diagonale qui va du sommet de cette montagne jusqu'au sommet du mont Hiei, c'est-à-dire une droite à $45^{\circ}$ qui monte de l'angle sud-ouest vers le coin nord-est du bassin, on remarque alors que cette montagne se dressait dans l'angle sud-ouest de la capitale, hitsuji-saru 坤 selon la rose des vents sino-japonaise, une direction dite ura kimon 裏鬼門, 《 opposée à la porte des mânes ». Elle faisait ainsi face à la « porte des mânes », kimon 鬼門, la direction nord-est (ushi-tora艮, 丑寅), « direction qui est néfaste en permanence et pour tout le monde », comme le notait Bernard Frank (†1996) dans son Kata-imi et Kata-tagae (1958 [1998]). Selon ce dispositif, le sanctuaire Iwashimizu Hachimangû 石清水八幡宮, bâti à proximité de la roche sacrée, gardait la direction du sud-ouest de la capitale, alors que le palais impérial était implanté de manière à être dans l'axe nord-est du mont Hiei 比㕡山, qui en assurait la protection grâce à la fondation en son sommet de l'Enryakuji 延暦寺, le grand monastère de l'école Tendai 天台宗 devenu temple protecteur de l'État dès le IX ${ }^{\mathrm{e}}$ siècle. Le mont Hiei se dresse lui-même en vis-à-vis du mont Atago 愛宕山, siège du grand dieu Atago (Atago daigongen 愛宕大権現), un dieu qui possède les traits caractéristiques des divinités protectrices des chemins (dôsojin 道祖神) et qui, situé de l'autre côté du bassin selon un axe horizontal est-ouest, était supposé veiller sur la direction nord-est (inu- $i$ 乾, 戌亥) du palais impérial. Les monts Hiei et Atago sont les deux points culminants du bassin de Kyôto.

La cité impériale était également placée sous la protection d'un autre ensemble, les quatre sanctuaires du Grand général, Daishôgun 大将軍. Selon la doctrine de la Voie du Yin et du Yang, Daishôgun est l'esprit de la planète Vénus; il opère une révolution complexe de telle sorte qu'il occupe tour à tour l'un des quatre secteurs $(\mathrm{N}, \mathrm{S}, \mathrm{E}$, O) de l'espace, ce que symbolisaient les quatre sanctuaires dressés aux quatre orients du bassin de Kyôto. La tradition du sanctuaire Daishôgun Hachi-sha 大将軍八社 mentionne le transfert de ce lieu de culte depuis Kasuga 春日, son lieu d'origine, dans la plaine du Yamato. D'après le Yamashiro meishô-shi 山城名勝志 (Annales des sites pittoresques du Yamashiro, 1705, fasc. $7: 346$ ), les quatre sanctuaires du Grand général étaient le Daishôgun Hachi-sha 大将軍八社 aux portes du Daitokuji 大徳寺 pour la direction nord, le sanctuaire Fujinomori jinja 藤森神社 pour la direction sud, le sanctuaire Daishôgun 大将軍社 à proximité du hameau d'Okazaki 岡崎 pour la direction est et celui du hameau Daishôgun 大将軍村 pour la direction ouest. Par ce dispositif, les Quatre secteurs de l'espace et les Quatre portes situées aux coins (NE, $\mathrm{SE}, \mathrm{NO}, \mathrm{SO}$ ) de la planche terrestre se trouvaient ainsi sous la garde bienveillante des divinités.

Les montagnes, les éminences rocheuses et les divinités gardiennes de l'espace qui y siégeaient ne constituaient pas les seuls éléments de croyances relatives à la protection de la capitale. Des cérémonies d'apaisement des âmes des morts étaient célébrées sur les landes sauvages, no 野, situées à l'entour de la capitale, où l'on pratiquait les crémations et où l'on enterrait les cendres des défunts. Ces landes étaient des lieux propices au contact avec l'autre monde. À l'époque de la fondation de Heian, l'es- 
sentiel de l'espace compris entre l'agglomération urbaine et les montagnes alentour, soit sur une largeur de un à trois kilomètres selon la direction, formait une lande quasi inhabitée et inexploitée. Au cours de la première partie de la période de Heian, on attribua un nom aux terres sauvages qui étaient l'objet de déplacements impériaux ou constituaient un lieu de chasse du souverain. La chasse n'était pas considérée comme un simple divertissement, ni seulement comme un besoin de première nécessité. On pense qu'il s'agissait d'un rite de revitalisation, par le transfert dans son corps des forces magiques et pleines de vitalité des esprits des monts et des landes. La notion de no (lande) évoquait un espace non quotidien, inhabité et sauvage, qui prenait sens par le fait qu'il n'était pas souillé par la présence de l'homme. Depuis l'établissement de la capitale (et en partie jusqu'à la période moderne), ces landes sauvages ont matérialisé des zones frontières jouxtant un autre monde.

De ce point de vue, les frontières spirituelles de l'ancienne capitale étaient sans doute plus nombreuses, comme en témoignent de nombreux rites : michiae no matsuri 道饗祭 (célébrations des divinités des chemins), ekijinsai 疫神祭 (célébrations des divinités des maladies faites aux quatre points cardinaux), kikisai 鬼氣祭 (célébrations des démons et des miasmes) ou encore ôharae 大祓 (rites de la Grande purification) et tsuina no girei 追儺の儀礼 (cérémonie de renvoi des démons). Les célébrations des divinités des chemins, par exemple, permettaient aux habitants de Kyôto de bloquer l'intrusion dans la cité des esprits malfaisants responsables des épidémies. Selon les prescriptions fixées dans les Codes, il fallait accomplir ces rites aux quatre coins de la capitale deux fois l'an, à la sixième et à la douzième lune. Les quatre points reliés simultanément par l'action des rites devaient ainsi former une ligne de protection. Cette pratique perdure jusqu'à la période moderne, comme l'évoque un passage du Meiji tennô ki 明治天皇記 (Récit sur l'empereur Meiji, 1868, Meiji 1.2.28), dans lequel il est dit que des célébrations des divinités des chemins furent effectuées aux quatre portes de la ville à l'occasion de l'entrée dans Kyôto des délégations diplomatiques d'Angleterre, de France et de Hollande.

Après un large tour d'horizon sur l'organisation matérielle du plan de la capitale impériale, on en est arrivé à la conclusion que les principaux sites naturels sacrés, situés aux alentours de Kyôto, avaient été directement associés à la conception de la ville, en tant qu'éléments constitutifs de son tracé et de son paysage urbains. Tout au long de l'histoire de Kyôto, d'autres sites furent chargés de la protection de la capitale et les rites qui y étaient associés ont instauré eux aussi un rapport constant entre l'espace urbain du centre de la plaine et l'espace naturel des collines et des montagnes avoisinantes. Les fêtes de renvoi par le feu, okuribi 送り火, au cours desquelles les immenses feux de Daimonji 大文字 sont allumés chaque année depuis la fin du Moyen Âge sur plusieurs collines autour de Kyôto, témoignent de la vivacité de certaines de ces pratiques. Au-delà des seules limites de son tracé physique, Kyôto a fixé des frontières conceptuelles à son étendue géographique, juxtaposées en un ensemble de cercles concentriques : la montagne - la plaine, la nature sauvage - l'espace habité; la forêt - la lande - l'agglomération urbaine; le monde des divinités - le monde des morts - le monde des vivants. La définition de ces limites et le désir qu'elles perdurent ont imposé l'implantation de protections invisibles autour de la cité, suivant un chapelet de sites naturels à la topographie remarquable et habités d'un genius loci. Une 
divinité, tantôt issue du shintô, tantôt du bouddhisme, tantôt de la Voie du Yin et du Yang, a pu être invoquée pour assurer la protection de la cité impériale. Quelle que fût la direction vers laquelle ils se tournaient, les habitants de Kyôto ont eu, tout au long de leur histoire, le sentiment de lieux naturels habités par les dieux, à l'abri du faisceau des frontières protectrices des collines boisées du pourtour de la ville.

Ces conceptions, animées par un souci de protection du palais impérial, ont imprimé durablement leur marque dans l'urbanisme de Kyôto. Elles ont aussi façonné, dans un second temps, un ensemble de représentations paysagères particulièrement originales. Dans ce domaine, l'étude de documents tardifs ( $\mathrm{XV}^{\mathrm{e}}-\mathrm{XVIII}{ }^{\mathrm{e}}$ siècles) alliant l'inscription à l'image - peintures de paysage peintes sur les parois des palais, vues à vol d'oiseau de la capitale montées en paravent pour orner les salles de réception et représentations cartographiques de la capitale - des documents qui donnent à voir la ville en un microcosme, fera l'objet de prochaines conférences, afin de montrer aux étudiants comment ces ensembles paysagers, restés vivaces tout au long de l'histoire de Kyôto, ont été conservés, reproduits et transmis, pour devenir en fin de compte des éléments de mémoire collective à la source d'un art du paysage original. 